\title{
DESENVOLVIMENTO E CALIBRAÇÃO DE UM TENSIÔMETRO ELETRÔNICO DE LEITURA AUTOMÁTICA ${ }^{1}$
}

\author{
ADUNIAS S. TEIXEIRA ${ }^{2}$, SILVANA L. COELHO ${ }^{3}$
}

RESUMO: O presente trabalho apresenta o desenvolvimento e calibração de tensiômetro de leitura automática, sendo o tensiômetro de mercúrio utilizado como padrão de comparação. Os ensaios foram conduzidos no Laboratório de Mecânica e Eletrônica do Departamento de Engenharia Agrícola da UFC. Esse equipamento difere do tensiômetro tradicional por substituir o manômetro de mercúrio por sensor de pressão. Tal dispositivo gera uma saída com valor de até no máximo 4,5 volts. O tensiômetro eletrônico é conectado a uma placa de aquisição de dados (DAQ) contendo um microprocessador, conversor analógico/digital (ADC) e saída serial, sendo tal placa ligada a um microcomputador. A bancada de ensaio constituiu-se de três caixas plásticas preenchidas com solo de textura francoarenosa. Os dados foram coletados durante um mês e as equações geradas foram do tipo Count $=$ offset $+\mathrm{a} \Psi_{\mathrm{m}}$, em que $\Psi_{\mathrm{m}}$ representa o potencial matricial fornecido pelo tensiômetro de mercúrio $(\mathrm{kPa})$ e count a saída digial do ADC, com valores adimensionais de 0 a 4.095. Foram obtidos os valores máximo e mínimo de offset de 348,572 e 261,026, e de coeficiente angular de $36,675 \mathrm{kPa}^{-1}$ e $34,421 \mathrm{kPa}^{-1}$. Os resultados da regressão linear simples indicaram a existência de regressão a menos de $0,1 \%$ de significância e valores de coeficientes de correlação nunca inferiores a 0,9994 .

PALAVRAS-CHAVE: sensor de pressão, automação, potencial matricial.

\section{DEVELOPMENT AND CALIBRATION OF AN ELECTRONIC TENSIOMETER FOR AUTOMATIC READING}

\begin{abstract}
This paper presents the development and calibration of a tensiometer for automatic reading where mercury has been replaced by an electronic pressure sensor called the electronic tensiometer. The conventional mercury tensiometer was used as a standard for calibration. Calibration trials were conducted at the Mechanics and Electronics Laboratory of the Department of Agricultural Engineering at Ceará Federal University, Brazil. The electronic tensiometer outputs a maximum 4.5 volts and is connected to a data acquisition system (DAQ) composed of a microprocessor, a 12-bit analog to digital converter and a serial port. The DAQ outputs to a microcomputer via a serial port. The trial bench was made of three hard plastic boxes filled with sandy-loam soil. Calibration trials have run for a month and linear calibration equations of the form Count $=$ offset $+a \psi_{m}$, were obtained, where $\Psi_{\mathrm{m}}$ represents the soil-water matric potential read at the conventional mercury tensiometer $(\mathrm{kPa})$ and count, the digital output from the electronic tensiometer, with dimensionless values ranging from 0 to 4,095. Maximum and minimum values of 348.572 and 261.026 were obtained for the offset, and $36.675 \mathrm{kPa}^{-1}$ e $34.421 \mathrm{kPa}^{-1}$ for the angular coefficient. Statistical analysis on the offset and coefficients of the calibration equations indicated that exists a significance confidence lower than $0.1 \%$ and correlation coefficients of at least 0.9994 for any of the nine sensors tested.
\end{abstract}

KEYWORDS: pressure sensor, automation, matric potential.

\footnotetext{
${ }_{2}^{1}$ Apoio financeiro do CNPq - CTHidro e Funcap.

${ }^{2}$ Prof. Adjunto, Ph.D., Departamento de Engenharia Agrícola, UFC, Fortaleza - CE, Fone: (0XX85) 288.9760, adunias@ufc.br

${ }^{3}$ Eng $^{\mathrm{a}}$ Agrônoma, Mestre em Irrigação e Drenagem, UFC, Fortaleza - CE, Fone: (0XX85) 288.9754, silvanacoelho@ dena.ufc.br

Recebido pelo Conselho Editorial em: 3-6-2003

Aprovado pelo Conselho Editorial em: 22-3-2005
}

Eng. Agríc., Jaboticabal, v.25, n.2, p.367-376, maio/ago. 2005 


\section{INTRODUÇÃO}

Diversos métodos podem ser utilizados para medir a umidade do solo visando a determinar a disponibilidade da água às plantas de forma a permitir seu pleno desenvolvimento, minimizando o gasto de energia na absorção de água e nutrientes. Os principais métodos são: o padrão da estufa, que fornece de forma direta os valores de umidade no solo, e os indiretos, que tomam como base medidas da moderação de nêutrons, da resistência do solo à passagem de uma corrente elétrica, da constante dielétrica do solo e da tensão da água no solo. Deve-se lembrar que tais métodos que fornecem valores de umidade de forma indireta, necessitam de calibração. Esses métodos vêm evoluindo ao longo dos tempos, tendo por objetivo sua adaptação às atuais tecnologias.

A versão do tensiômetro proposta por Gardner, em 1922, vem sofrendo modificações visando a, sobretudo, facilitar a instalação, operação e manutenção do equipamento no campo, melhorar o tempo de resposta, aumentar a sensibilidade, facilitar a leitura automática dos dados e realizar o controle automático de sistemas de irrigação. Nesse sentido, o tensiômetro aneróide, que toma como base leituras de pressão da câmara de ar formada na parte superior do equipamento, foi desenvolvido. Esse instrumento permitiu evitar os riscos de contaminação causada pelo manômetro de mercúrio, porém a leitura é feita de forma manual.

O transdutor de pressão, nome dado aos sistemas que transformam sinais de pressão em outros tipos, vem sendo usado acoplado a tensiômetros para monitorar a umidade no solo em campo e em laboratório. Os medidores eletrônicos substituem os medidores do tipo manômetro de mercúrio, Bourdon e vacuômetro, o que possibilita a coleta automática de coleta de dados por meio da utilização de computador ou datalogger (MOTOROLA, 1997). Segundo WANG et al.(1998), transdutores sensíveis à pressão e sistemas multicanais de dados habilitaram o uso de tensiômetros em várias aplicações.

O tensiômetro convencional de mercúrio vem sendo utilizado como padrão de calibração de sensores de pressão por diversos autores, tais como CAMARGO et al. (1982), VILLA NOVA et al. (1992) e ASSIS JÚNIOR (1995), em trabalhos com tensiômetro de câmara de ar, e MARTHALER (1983) quando do desenvolvimento do tensímetro.

O presente trabalho teve como objetivo o desenvolvimento, calibração e validação de um dispositivo do tipo tensiométrico de leitura automática, aqui denominado de "tensiômetro eletrônico", para o monitoramento do potencial de água no solo.

\section{MATERIAL E MÉTODOS}

\section{Tensiômetro eletrônico}

Os componentes do tensiômetro eletrônico encontram-se especificados na Figura 1a, na qual se podem observar o dispositivo eletrônico (1) e a cápsula porosa em uma extremidade (2). Na Figura 1b, apresenta-se a ampliação do circuito eletrônico contendo o sensor de temperatura (12), o sensor de pressão diferencial (4), a saída (5) conectada à cápsula porosa (2) pelo microtubo (9), e a saída (6) conectada diretamente à atmosfera. Também são apresentados o tubo de PVC (7), cap de PVC (8), peão e O-ring (10) que formam a parte hidráulica do sistema onde a água (11) é contida.

\section{Teste da cápsula}

Testes de condutância e de pressão de borbulhamento foram realizados para um conjunto de 16 cápsulas porosas. Os testes foram conduzidos no Laboratório de Física de Solos do Departamento de Engenharia Agrícola - DENA, da Universidade Federal do Ceará - UFC, seguindo o procedimento preconizado por LIBARDI (1999). A realização do teste de condutância teve o objetivo de agrupar as cápsulas, enquanto o teste de pressão de borbulhamento teve o objetivo de descartar cápsulas com 
pressões de borbulhamento inferiores a 1 atm. Para o teste de condutância, as cápsulas porosas foram submetidas às pressões entre $25,3 \mathrm{kPa}(0,25 \mathrm{~atm})$ e $152 \mathrm{kPa}(1,5 \mathrm{~atm})$ em intervalos de $25,3 \mathrm{kPa}$ (0,25 atm), com cada cápsula submetida a três repetições.

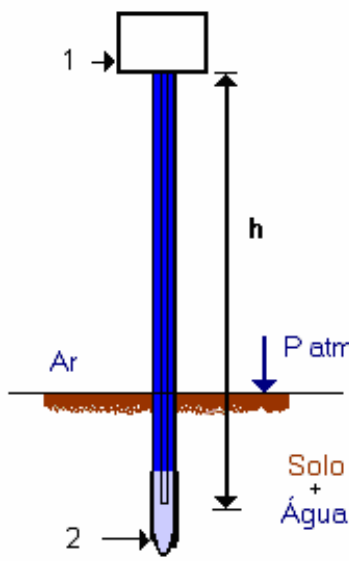

(a)

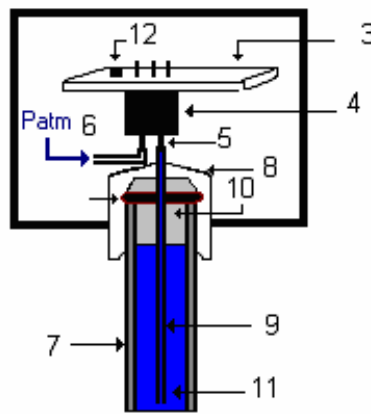

(b)

FIGURA 1. Layout do tensiômetro eletrônico (a) e disposição do circuito eletrônico (b).

\section{Circuito elétrico}

A placa de circuito com os componentes do tensiômetro eletrônico pode ser visualizada na Figura 2, na qual se encontram destacados seus componentes, tais como, a fonte de alimentação (1), regulador de voltagem (2), led (3), sensor de temperatura (4) e o sensor de pressão (5). Também fazem parte do circuito capacitores e resistores, os quais têm por finalidade a filtragem da voltagem de alimentação, visando a eliminar ruídos do sistema. O regulador de voltagem (2) tem a função de manter a alimentação constante em 5 volts.

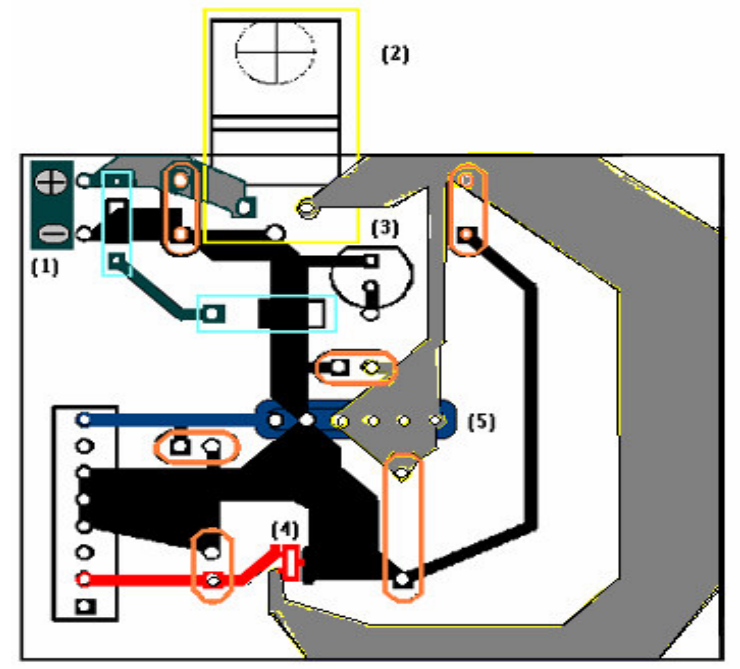

FIGURA 2. Placa de circuito impressa e seus componentes.

\section{Sensores}

Pré-testes em diversos modelos de sensores de pressão serviram de base para indicar a seleção do sensor MPX5100 DP, fabricado pela Motorola, como sensor de pressão diferencial (Figura 3a). Já para o sensor de temperatura, foi utilizado o modelo FM51, fabricado pela Fairchild semicondutores 
(Figura 3b), devido, sobretudo, à disponibilidade, ser compacto, alimentação de 5 volts e precisão esperada menor do que $1{ }^{\circ} \mathrm{C}$.

Os sensores de pressão têm como elemento sensível piezorresistores de silício monolítico, que geram voltagem de saída que varia conforme a pressão aplicada. O elemento resistivo, que constitui o medidor de pressão, é uma implantação de íon em um fino diafragma de silício. A pressão aplicada sobre o diafragma resulta em variação de resistência no medidor de pressão que, por conseguinte, promove variação na voltagem de saída diretamente proporcional à pressão aplicada (MOTOROLA, 2001). Ainda segundo o fabricante, o sensor de pressão não sofre efeito de temperatura e opera com diferenças de pressão que podem variar de $-100 \mathrm{a}+100 \mathrm{kPa}$, com voltagem típica de suprimento de 5 volts. A sensibilidade do sensor de pressão, segundo características operacionais, é de $45 \mathrm{mV}(\mathrm{kPa})^{-1}$.

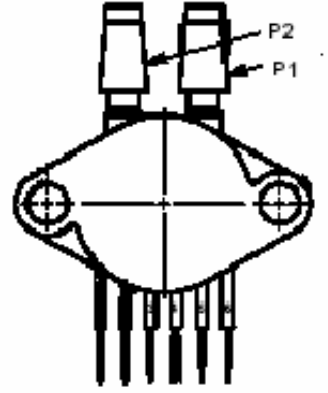

(a)

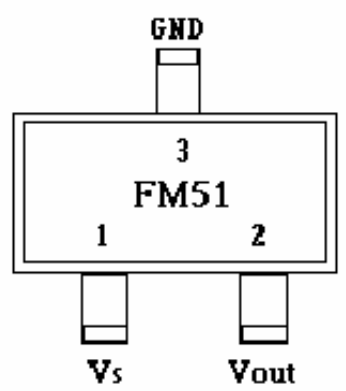

(b)

FIGURA 3. Layout dos sensores de pressão MPX5100D (a) e de temperatura FM51 (b).

A voltagem máxima na saída do sensor de pressão é de 4,5 volts e no offset $2 \mathrm{mV}$, para diferenças de pressões respectivas de $100 \mathrm{kPa}$ e $0 \mathrm{kPa}$. Assim, para um sistema de aquisição de dados com conversor analógico digital (ADC) de 12 bits, tem-se um valor máximo de counts na base decimal de $4.095\left(2^{12}-1\right)$. O valor do offset equivale a 2 counts (resultado inteiro de $(0,002 / 5) 4.095$, e o valor máximo de voltagem equivale a 3.686 counts (resultado inteiro de $(4,5 / 5) 4095$, o que resulta em uma faixa de 3.684 counts para uma pressão de $100 \mathrm{kPa}$. Portanto, para a configuração do sistema aqui apresentado, tem-se uma sensibilidade potencial de 0,001221 volts por count $((4,5-0,002) / 3.684)$ ou 36,84 counts por $\mathrm{kPa}$, o que equivale a 3,57 counts por $\mathrm{cm} \mathrm{H}_{2} \mathrm{O}$ e resulta em precisão potencial de $2,8 \mathrm{~mm} \mathrm{H}_{2} \mathrm{O}$.

O FM 51 é um sensor de temperatura de alta precisão, baixa voltagem (2,7 a $6 \mathrm{~V})$, sendo uma solução viável em termos de custo quando aplicado no monitoramento de temperatura. A relação voltagem de saída versus temperatura é extremamente linear com resposta de $10 \mathrm{mV} /{ }^{\circ} \mathrm{C}$. $\mathrm{Na}$ faixa de temperatura de -40 a $125{ }^{\circ} \mathrm{C}$, sua exatidão é de $+/-1{ }^{\circ} \mathrm{C}$ e de $+/-0,5{ }^{\circ} \mathrm{C}$ em temperatura ambiente. Segundo o fabricante, não há necessidade de calibração, pois todas as partes foram calibradas e testadas durante o processo de montagem. Contudo, devido às especificidades do circuito eletrônico, cada sensor de temperatura foi calibrado individualmente, conforme ARAÚJO et al. (2003), para fornecer de 0,5 a 1 volt entre 0 e $50{ }^{\circ} \mathrm{C}$.

Considerando que o total da solução do solo, nos locais próximos à cápsula porosa, esteja em equilíbrio com o potencial dentro do equipamento e, levando-se em consideração as particularidades do sensor, como o fato de se tratar de um sensor do tipo diferencial, é possível desenvolver as relações de pressão.

Chamando $\mathrm{P}_{1}$ e $\mathrm{P}_{2}$ as pressões atuando nas duas saídas (6) e (5) do sensor apresentadas na Figura $1 \mathrm{~b}$, a diferença de pressão entre os dois pontos $\Delta \mathrm{P}=\mathrm{P}_{2}-\mathrm{P}_{1}$, determina a resposta do sensor em volts, isto é: Vout $=f(\Delta \mathrm{P})=f\left(\mathrm{P}_{2}-\mathrm{P}_{1}\right)$, sendo Vout a resposta do sensor em volts. 
Analisando o esquema de instalação do tensiômetro eletrônico (Figuras 1a e 1b), é possível identificar que: $\mathrm{P}_{1}=\mathrm{P}_{\mathrm{atm}}$ e $\mathrm{P}_{2}=\mathrm{P}_{\mathrm{atm}}+\gamma_{\mathrm{H} 2 \mathrm{O}} \mathrm{h}+\Psi_{\mathrm{m}}$, ou seja, $\Delta \mathrm{P}=+\gamma_{\mathrm{H} 2 \mathrm{O}} \mathrm{h}+\Psi_{\mathrm{m}}$.

Assim, a resposta do sensor apresenta dependência do potencial matricial, das características do sensor em si, bem como do comprimento da coluna de água no tubo de PVC do corpo do sensor, $\mathrm{h}$.

\section{Bancada de ensaios}

Os ensaios para fins de calibração do tensiômetro eletrônico foram conduzidos no Laboratório de Mecânica e Eletrônica do Departamento de Engenharia Agrícola da UFC. A bancada de ensaios consta de um conjunto de três caixas plásticas medindo $0,34 \mathrm{~m}$ x $0,53 \mathrm{~m} \times 0,30 \mathrm{~m}$, fechadas nas laterais e com a face inferior perfurada para permitir a drenagem do solo. Essas caixas receberam a denominação de caixas-teste.

O solo utilizado no preenchimento das caixas-teste é de textura franco-arenosa, com $5 \%$ de areia grossa, $61 \%$ de areia fina, $24 \%$ de silte e $10 \%$ de argila. O solo coletado foi seco ao ar, destorroado e peneirado em malha de $2 \mathrm{~mm}$ de diâmetro. As caixas foram preenchidas, primeiramente, com uma camada de brita e areia grossa de diâmetro superior a $2 \mathrm{~mm}$ e densidade $1.300 \mathrm{~kg} \mathrm{~m}^{-3}$. No topo da areia, foram depositadas camadas de solo de forma a manter a densidade do solo de aproximadamente $1.400 \mathrm{~kg} \mathrm{~m}^{-3}$ (Figura 4a).

Após alguns dias do preenchimento das caixas, o solo foi saturado com aproximadamente $20 \mathrm{~L}$ de água. Após a drenagem do excesso de água, os tensiômetros foram instalados à profundidade de 0,10 m. Quatro tensiômetros foram instalados por caixa, sendo um de mercúrio e três eletrônicos. No total, foram utilizadas três caixas denominadas de A, B e C. A distribuição dos tensiômetros nas caixas foi feita de forma aleatória (Figura 4b).

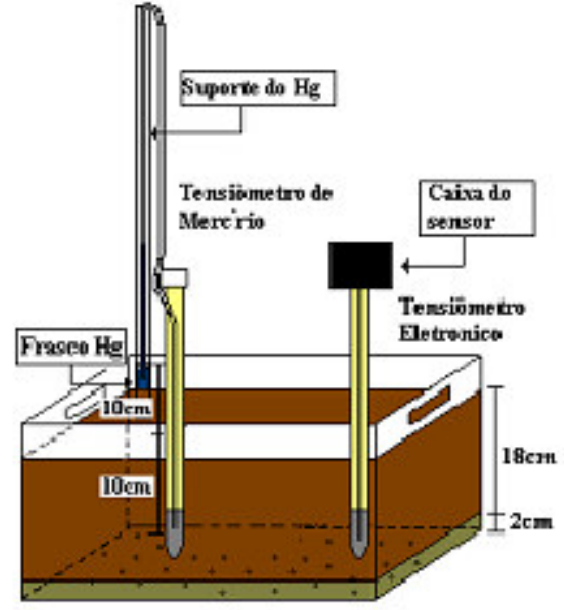

(a)

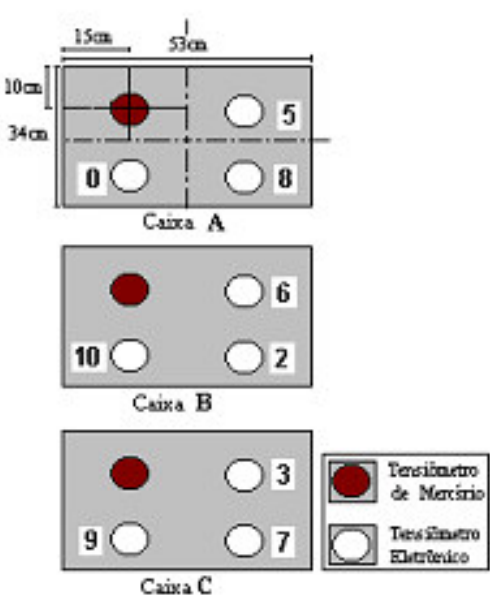

(b)

FIGURA 4. Esquema da distribuição dos sensores (a) e disposição dos tensiômetros na caixa-teste (b).

A fluxagem dos tensiômetros foi efetuada de modo distinto para os dois tipos de instrumentos. Para o tensiômetro de mercúrio, a substituição do ar no interior do microtubo por água destilada foi feita utilizando-se de uma seringa de $50 \mathrm{~mL}$. Já o tensiômetro eletrônico, por apresentar modificações em sua estrutura, requer modificações no processo de instalação. O microtubo que interliga o sensor à cápsula foi preenchido com água destilada com a ajuda de uma seringa de $10 \mathrm{~mL}$, com agulha presa a um tubo capilar. $\mathrm{O}$ tubo capilar foi inserido através do microtubo até o sensor de pressão e a seringa pressionada para que a água preenchesse o microtubo de baixo para cima. Em seguida, o tubo de PVC foi preenchido com água destilada e o circuito eletrônico preso no topo do tubo de PVC. 
Vale ressaltar que o tensiômetro eletrônico pode ser interfaciado com qualquer datalogger ou placa de controle disponível. No esquema experimental, o sistema de aquisição de dados (DAQ) constituiu-se de um conversor analógico/digital (ADC) de 12 bits e 11 canais, um microcontrolador (AMD 188), saída serial e microcomputador. Um programa em linguagem $C$ funcionou como sistema operacional para o microcontrolador. O DAQ transmitia os dados dos sensores de temperatura e pressão em valores na base 10 (counts) para o microcomputador portátil, utilizando a interface serial. Os dados eram apresentados na tela do computador em tempo real a uma frequiência de $2 \mathrm{~Hz}$, e gravados em arquivo de texto para posterior processamento (Figura 5). Dez canais foram utilizados para receber os dados enviados pelos sensores, sendo um ligado a um sensor de temperatura e nove ligados a sensores de pressão.

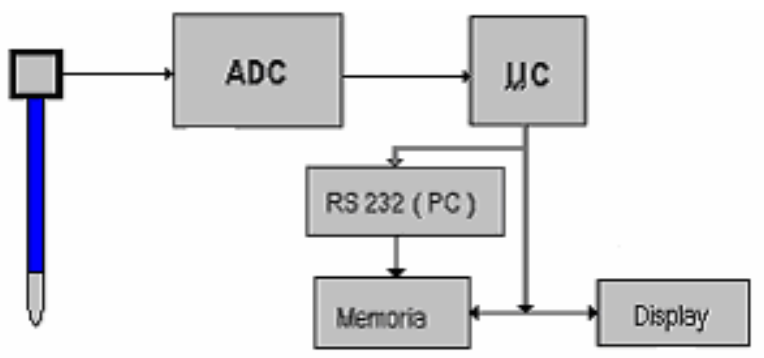

FIGURA 5. Representação esquemática do sistema de aquisição de dados.

\section{Calibração do sensor de pressão}

A calibração consta da determinação da relação funcional entre as tensões obtidas pelo tensiômetro de mercúrio, utilizado como padrão, e a média de counts obtidas no DAQ dos tensiômetros eletrônicos.

As leituras no tensiômetro de mercúrio foram realizadas, em sua maioria, no início da manhã, ao meio-dia e no final da tarde, todos os dias, sem exceção, durante 32 dias. Para a obtenção dos valores médios dos counts, utilizou-se do DAQ e de um programa em linguagem $\mathrm{C}$ que fazia a média dos valores de counts a cada dez minutos, obtida dos valores tomados cinco minutos antes e cinco minutos após a leitura no tensiômetro de mercúrio.

O método de minimização da soma do quadrado do resíduo foi utilizado para desenvolver a relação funcional entre os valores de counts e o potencial matricial, tendo a análise de variância da regressão sido utilizada para testar as hipóteses de validação dos coeficientes da regressão.

\section{RESULTADOS E DISCUSSÃO}

\section{Testes na cápsula porosa}

Para a pressão de 101,325 kPa (1 atm), o valor médio de condutância das 16 cápsulas foi de $1,9 \times 10^{-8} \mathrm{~m}^{2} \mathrm{~s}^{-1}$, sendo superior ao encontrado por GONDIM (1998), de 1,54 x 10 $0^{-8} \mathrm{~m}^{2} \mathrm{~s}^{-1}$. Essa diferença deve-se à maior superfície das cápsulas utilizadas no experimento. Todas as cápsulas apresentaram a pressão de borbulhamento superior a 101,325 kPa. Pode-se, então, afirmar que se trata de um lote de cápsulas de excelente qualidade, com altos valores de condutância, bem acima do valor definido como suficiente, segundo CASSEL \& KLUTE (1986) de $10^{-9} \mathrm{~m}^{2} \mathrm{~s}^{-1}$, e pressão de borbulhamento acima de 101,325 kPa, valor mínimo limitante estipulado por LIBARDI (1999).

Das cápsulas testadas, somente doze foram utilizadas na fabricação dos tensiômetros, tendo sido selecionadas as cápsulas de número $3 ; 4 ; 5 ; 8 ; 11 ; 13 ; 14 ; 16$ e 17 por terem apresentado maior uniformidade nos valores de condutância (Figura 6) e maiores valores de pressão de borbulhamento. 


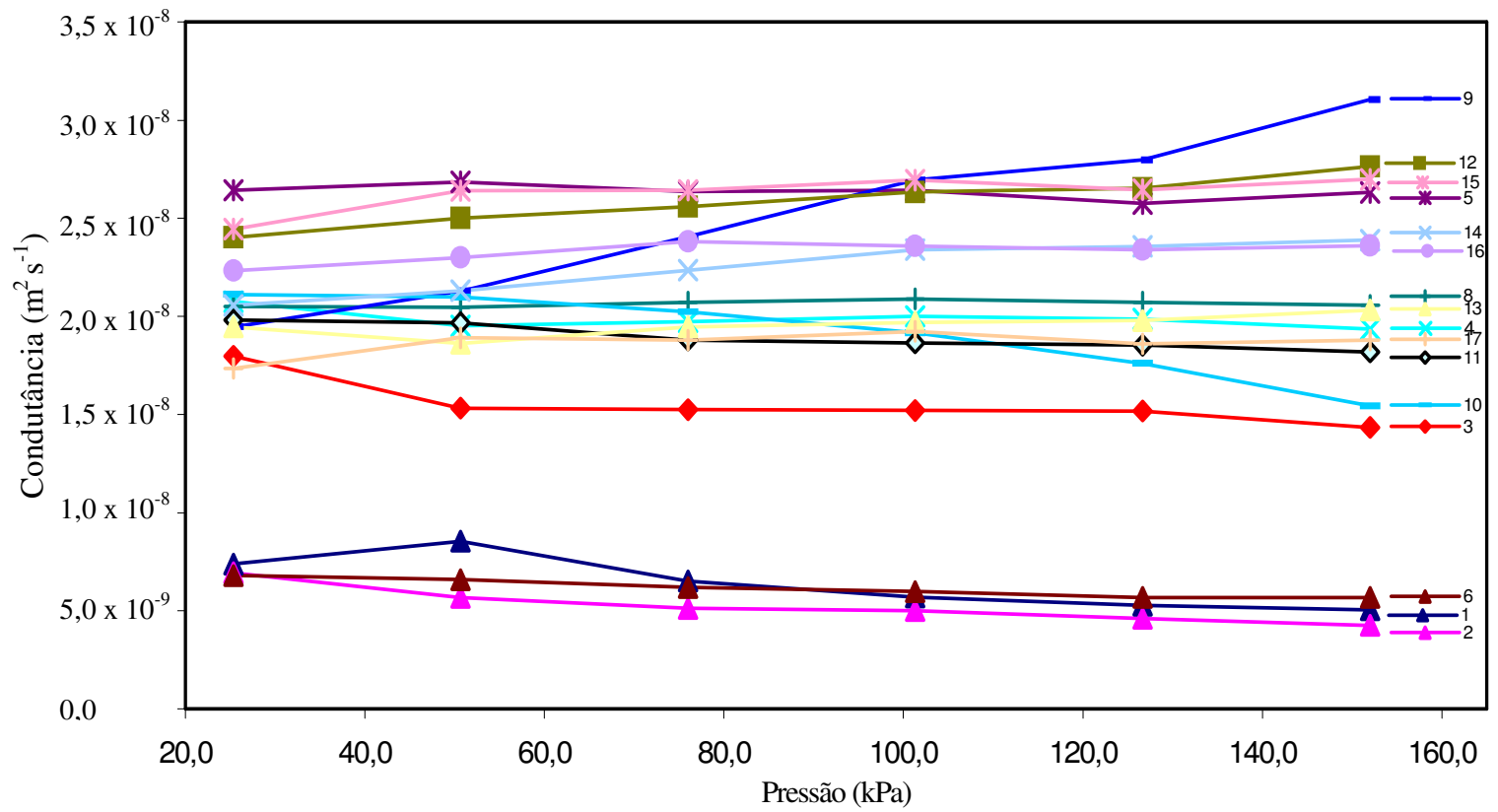

FIGURA 6. Condutância das cápsulas porosas.

\section{Calibração do tensiômetro eletrônico}

As leituras dos nove tensiômetros eletrônicos (em counts) foram comparadas com os valores de potencial matricial (em $\mathrm{kPa}$ ) obtidos pelos tensiômetros de mercúrio instalados nas respectivas caixasteste (Figura 4a). A caixa-teste A apresentou problemas no tensiômetro de mercúrio, sendo necessária a "fluxagem" do dispositivo quando o potencial se aproximava de $25,5 \mathrm{kPa}\left(260 \mathrm{~cm} \mathrm{H}_{2} \mathrm{O}\right)$. Os valores de potencial matricial maiores que duas vezes o desvio-padrão, considerados discrepantes (outliers), foram excluídos da análise. Os sensores da caixa-teste B não apresentaram problemas aparentes no decorrer do período. Na caixa-teste $\mathrm{C}$, o sensor 9 apresentou problema a partir das leituras próximas a 2.000 counts, o que pode ter sido causado pelo rompimento do selo de pressão interna do dispositivo, tendo os demais sensores da respectiva caixa-teste funcionado sem problemas.

As equações de calibração do tipo Count $=$ offset $+a \psi_{m}$, obtidas por regressão linear para cada sensor, são apresentadas na Tabela 1 , em que $\Psi_{\mathrm{m}}$ representa o valor absoluto do potencial matricial em $\mathrm{kPa} ; \mathrm{t}_{\mathrm{cal}}$ e $\mathrm{t}_{\mathrm{tab}}$, os parâmetros respectivos do teste $\mathrm{t}$ para valores calculados e tabelados; $\rho$ o nível de significância (decimal) mínimo para rejeitar a hipótese da nulidade do coeficiente $\boldsymbol{a}\left(\mathrm{kPa}^{-1}\right)$ e do offset da regressão. Observa-se, na Tabela 1, que os coeficientes de correlação foram bastante elevados, apresentando valores nunca inferiores a 0,9994. Os testes nos parâmetros da regressão indicam a existência da regressão em níveis de significância inferiores a $0,1 \%$.

TABELA 1. Parâmetros das equações de calibração dos tensiômetros eletrônicos.

\begin{tabular}{|c|c|c|c|c|c|c|c|c|c|}
\hline \multirow{2}{*}{ Sensor } & \multicolumn{4}{|c|}{ Offset } & \multicolumn{4}{|c|}{ Coeficiente $\boldsymbol{a}\left(\mathrm{kPa}^{-1}\right)$} & \multirow{2}{*}{$\mathrm{R}^{2}$} \\
\hline & Valor & $\mathrm{t}_{\mathrm{cal}}$ & $t_{\text {tab }}$ & $\rho$ & Valor & $\mathrm{t}_{\mathrm{cal}}$ & $\mathrm{t}_{\mathrm{tab}}$ & $\rho$ & \\
\hline 0 & 336,730 & 173,43 & 1,645 & $<0,001$ & 35,5926 & 547,67 & 1,645 & $<0,001$ & 0,9996 \\
\hline 5 & 317,128 & 158,26 & 1,645 & $<0,001$ & 35,7847 & 533,52 & 1,645 & $<0,001$ & 0,9995 \\
\hline 8 & 348,572 & 155,33 & 1,645 & $<0,001$ & 34,4213 & 458,26 & 1,645 & $<0,001$ & 0,9994 \\
\hline 2 & 258,388 & 212,12 & 1,645 & $<0,001$ & 35,0229 & 835,12 & 1,645 & $<0,001$ & 0,9996 \\
\hline 6 & 261,026 & 161,72 & 1,645 & $<0,001$ & 34,7839 & 625,94 & 1,645 & $<0,001$ & 0,9996 \\
\hline 10 & 261,570 & 283,33 & 1,645 & $<0,001$ & 35,2666 & 839,00 & 1,645 & $<0,001$ & 0,9998 \\
\hline 3 & 308,122 & 353,89 & 1,645 & $<0,001$ & 36,6753 & $1.134,64$ & 1,645 & $<0,001$ & 0,9999 \\
\hline 7 & 316,231 & 316,74 & 1,645 & $<0,001$ & 35,9732 & 970,56 & 1,645 & $<0,001$ & 0,9998 \\
\hline 9 & 320,451 & 338,96 & 1,645 & $<0,001$ & 35,4840 & 665,64 & 1,645 & $<0,001$ & 0,9997 \\
\hline
\end{tabular}




\section{Validação da equação de regressão}

Após a coleta de dados necessários para a obtenção da equação de calibração, os solos das caixas-teste foram irrigados novamente, dando-se início a um segundo ciclo de secagem que durou 12 dias. Nas Figuras 7; 8 e 9, apresentam-se as validações das equações de regressão para os sensores instalados nas caixas-teste $\mathrm{A}, \mathrm{B}$ e $\mathrm{C}$, respectivamente. Pode ser verificado que os sensores 0 e 8 da caixa-teste A superestimaram os valores de potenciais matriciais, com desvio absoluto médio de 1,61 e 2,8 kPa, respectivamente. Para o sensor 5, não foi possível coletar os dados devido a problemas com a vedação do equipamento. Os tensiômetros eletrônicos, pertencentes à caixa-teste $\mathrm{B}$, sempre superestimaram os valores de potenciais, contudo, com diferenças nunca superiores a 1,57; 1,60 e 1,50 $\mathrm{kPa}$, para os sensores 2; 6 e 10, respectivamente. $\mathrm{Na}$ caixa-teste $\mathrm{C}$, para potenciais entre aproximadamente - 16 e - $79 \mathrm{kPa}$, percebe-se que a maioria dos pontos encontram-se muito próximos à linha-base; somente a partir de potenciais acima de $-55 \mathrm{kPa}$ os valores encontram-se superestimados com desvio absoluto médio de 1,$11 ; 1,61$ e 3,08 kPa, para os sensores $3 ; 7$ e 10, respectivamente. $\mathrm{O}$ menor valor de coeficiente de correlação obtido na equação de validação que relaciona valores estimados com observados, foi de 0,9957 .

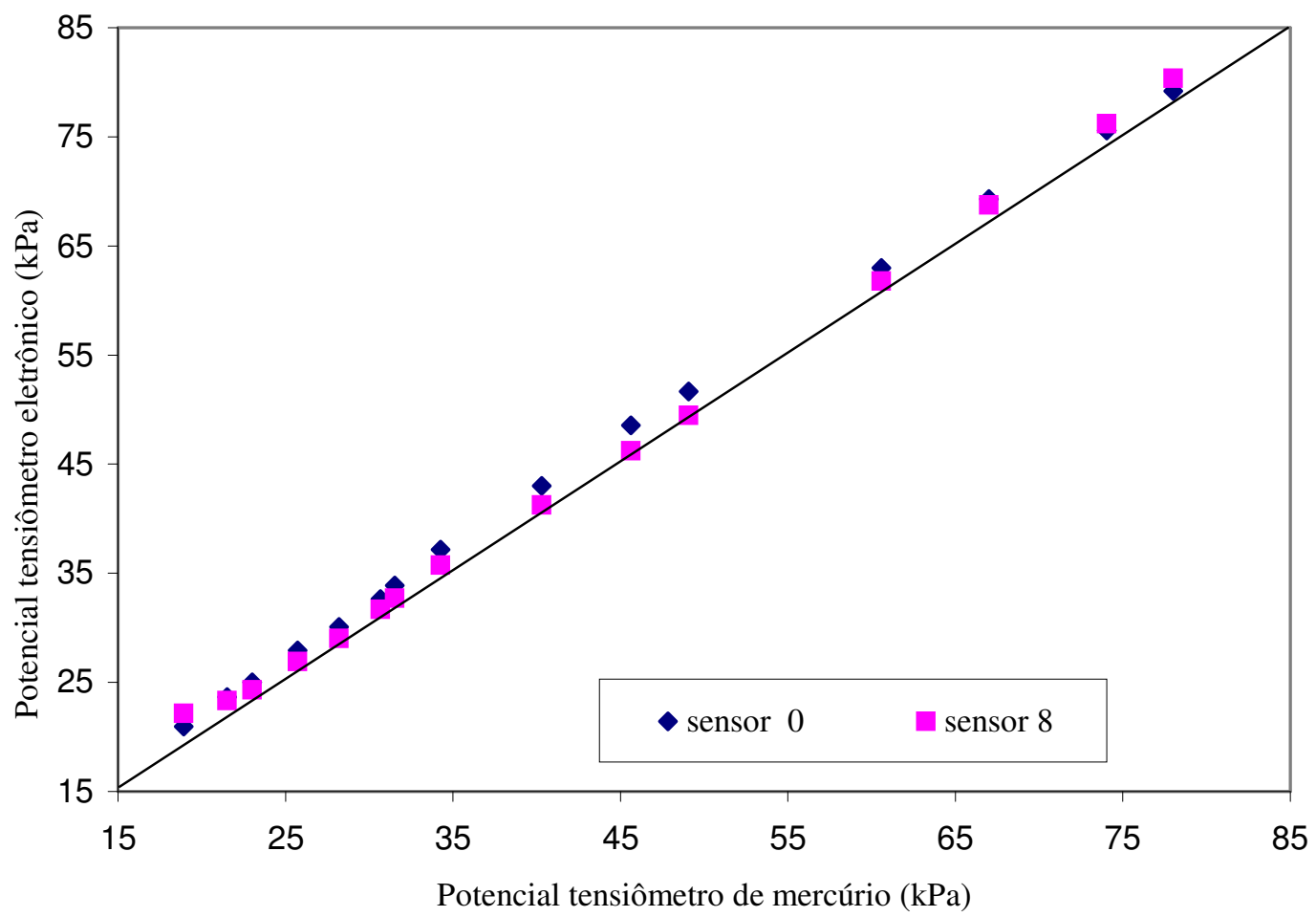

FIGURA 7. Validação da equação de regressão para os sensores da caixa-teste A. 


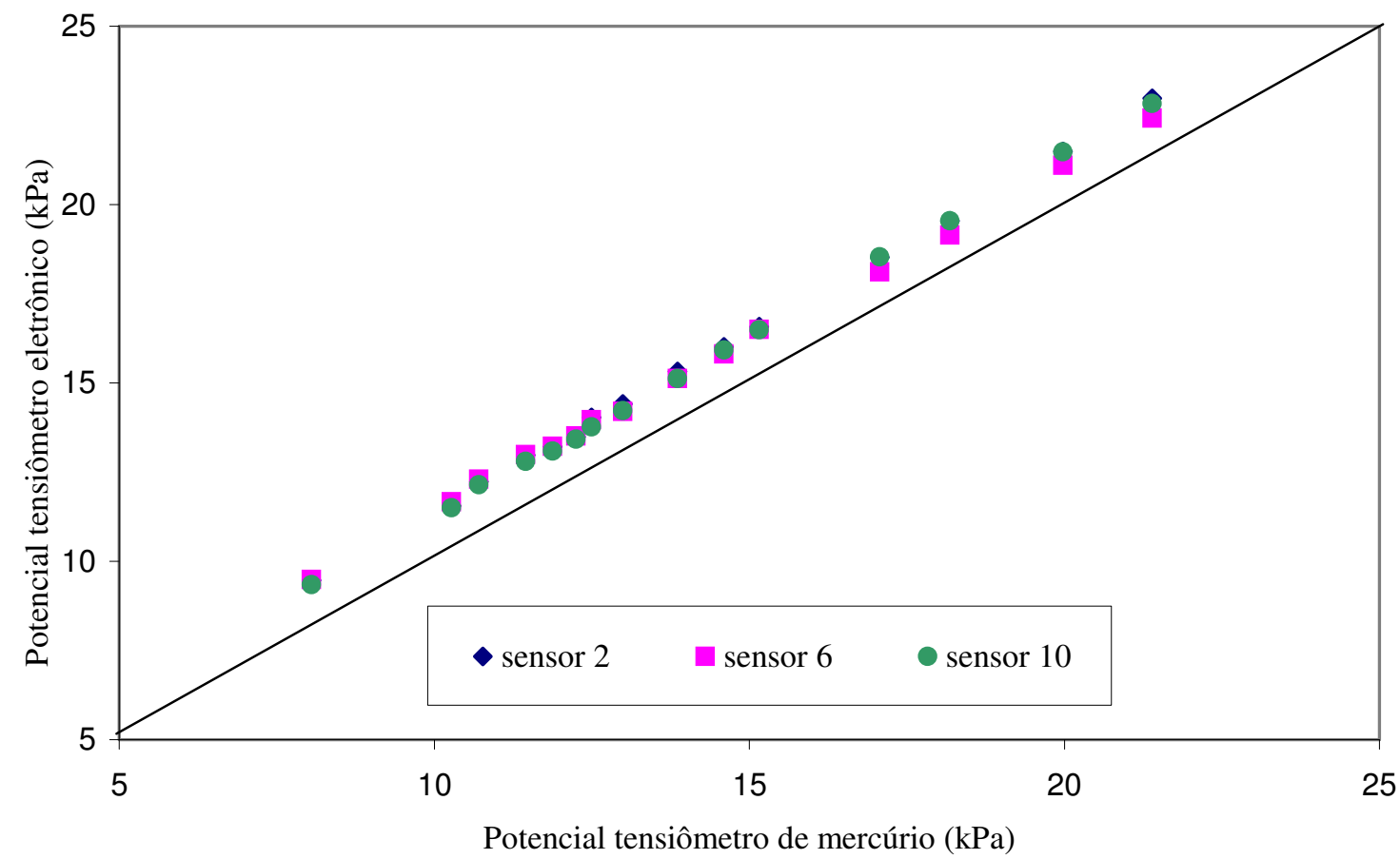

FIGURA 8. Validação da equação de regressão para os sensores da caixa-teste B.

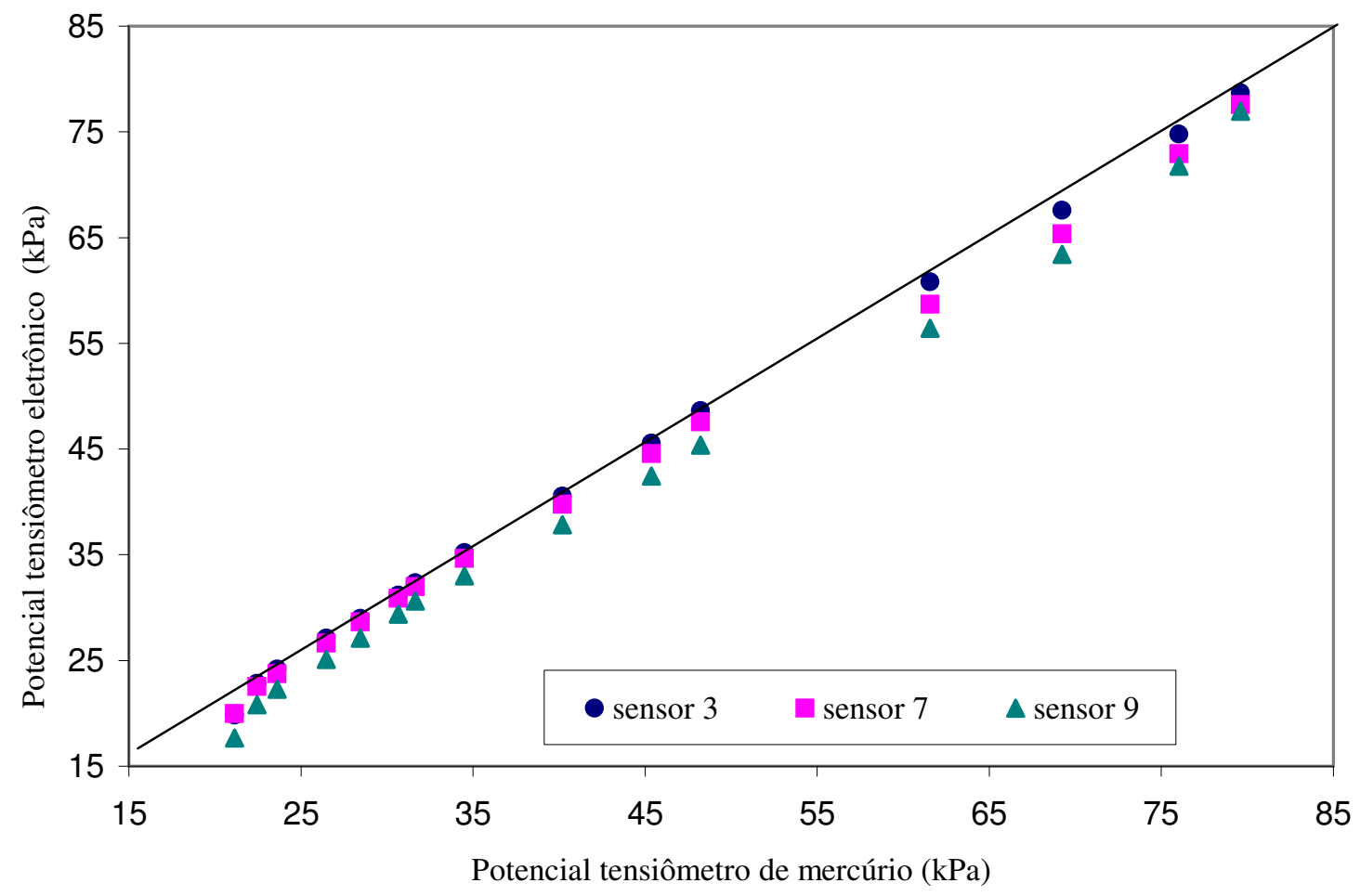

FIGURA 9. Validação da equação de regressão para os sensores da caixa-teste C. 


\section{CONCLUSÕES}

Os sensores de pressão apresentaram comportamento linear com testes de parâmetros de regressão, indicando a existência de regressão a $0,1 \%$ de significância, e os coeficientes de determinação apresentando valores superiores a 0,9970, para qualquer um dos tensiômetros eletrônicos testados.

A validação da função de resposta dos tensiômetros eletrônicos, realizada com valores de potenciais obtidos contra o tensiômetro de mercúrio, em um segundo ciclo de secamento do solo, demonstrou repetibilidade no comportamento do sensor, observada pelo coeficiente de determinação entre valores observados e calculados, cujo valor mínimo foi de 0,9957 para todos os tensiômetros eletrônicos avaliados.

\section{REFERENCIAS}

ARAÚJO, D.F.; COELHO, S.L.; TEIXEIRA, A.S. Calibração de um sensor de temperatura aplicado a um tensiômetro eletrônico de leitura automática. In: CONGRESSO BRASILEIRO DE

ENGENHARIA AGRÍCOLA, 32., 2003, Goiânia. Anais... Jaboticabal: Sociedade Brasileira de Engenharia Agrícola, 2003. 1 CD ROM.

ASSIS JÚNIOR, R.N. Análise de desenvolvimento do tensiômetro de câmara de ar na avaliação do potencial mátrico da água. 1995. 43 f. Tese (Doutorado em Física do Solo) - Escola Superior de Agricultura “Luiz de Queiroz”,Universidade de São Paulo, Piracicaba, 1995.

CAMARGO, A.P.; GROHMANN, F.; CAMARGO, M.B.P. Tensiômetro simples de leitura direta. Pesquisa Agropecuária Brasileira, Brasília, v.17, n.12, p.1763-72, dez. 1982.

CASSEL, D.K.; KLUTE, A. Water potential: tensiometry. In: . Methods of soil analysis. Madison: American Society of Agronomy, 1986. (Agronomy monograph, 9).

GONDIM, R.S.; AGUIAR, J.V.; COSTA, R.N.T. Avaliação de cápsulas porosas para tensiômetros através de testes de condutância hidráulica e pressão de borbulhamento. 1998. 52 f. Dissertação (Mestrado em Irrigação e Drenagem) - Universidade Federal do Ceará, Fortaleza, 1998.

LIBARDI, P.L.; REICHARDT, K. Tensiometria e variabilidade espacial em terra roxa estruturada. In: CONGRESSO BRASILEIRO DE CIÊNCIA DE SOLO, 21., 1987, Campinas. Anais... Campinas: Sociedade Brasileira de Ciência do Solo, 1987.

MARTHALER, H.P.; VOGERSANGER, W.; RICHARD, F.; WIERENGA, P.J. A pressure transducer for field tensiometers. Soil Science Society American Journal, Madison, v.47, n.4, p.624-7, 1983.

MOTOROLA, INC. Sensor devise data/handbook. $4^{\text {th }}$ ed. Phoenix: Motorola, 1997.

VILLA NOVA M.S.; VILLA NOVA, N.A.; OLIVEIRA, A.S.; REICHARDT, K. Performance and test of a direct reading "Air pocket" tensiometer. Soil Technology, Cremlingen, v.5, p.283-7, 1992.

WANG, D.;YATES, S. R.; ERNST, F.F. Determining soil hydraulic properties using tension infiltrometers, time domain reflectometry and tensiometers. Soil Science Society American Journal, Madison, v.62, p.318-25,1998. 